\title{
Severity and mortality of COVID-19 infection in HIV-infected individuals: Preliminary findings from Iran
}

\author{
Sana Eybpoosh ${ }^{1}$, Mahdi Afshari ${ }^{2 *} \mathbb{E}$, Ali-Akbar Haghdoost ${ }^{3}$, Parvin Afsar Kazerooni ${ }^{4}$, Mohammad Mehdi Gouya ${ }^{5}$, \\ Katayoon Tayeri ${ }^{6}$
}

Received: 7 Nov 2020

Published: 10 Mar 2021

\section{Abstract}

Background: Higher mortality due to coronavirus disease 2019 (COVID-19) is reported among some immunocompromised patients; however, the relation between immunosuppression due to HIV infection and severity of COVID-19 infection remains unclear. We aimed to investigate the severity and mortality of COVID-19 infection in HIV-infected patients.

Methods: This was a retrospective cohort study on all COVID-19 suspected and confirmed cases hospitlized in Iran between Febuary 19 (epidemic onset date) and April 8, 2020, whose data were recorded in the national database for Medical Care Monitoring Center. Hospitalized patients were followed from admittion to death/discharge. Patients' HIV status was recorded based on their self report. Logistic and Cox regression models were used to evaluate the association between HIV infection and the severity (according to the Glascow-Coma Scale situation, need for intubation and hypoxemia) and mortality of COVID-19 infection, respectively. Analyses were performed separately for COVID-19 suspected and confirmed cases.

Results: Out of 122206 severe acute respiratory infection (SARI) cases, 90 were HIV-positive (0.07\%), with a similar mean age (Pttest $=0.750)$ and distrubtion of gender (PChi-square $=0.887)$ and nationality (PChi-square $=0.202)$ as HIV-negative patients. A comparable proportion of HIV-positive and HIV-negative cases were tested for COVID-19 ( $\mathrm{p}=0.170)$; however, the frequency of positive results was lower among HIV-positives $(\mathrm{p}=0.038)$. The frequency of COVID-19 and HIV coinfection was lower than expected among confirmed cases (adjusted $\mathrm{OR}=0.54 ; 95 \%$ CI: 0.29-1.02) and suspected cases (adjusted OR $=0.68$; 95\% CI: 0.45 1.02), which means that the frequency of COVID-19 infection was lower among HIV-positive cases. HIV infection decreased the risk of death among confirmed (adjusted HR $=0.33 ; 95 \%$ CI: 0.05-2.32), suspected cases (adjusted HR=0.81; 95\% CI: 0.33-1.94), and among SARI cases (adjusted HR=0.73; 95\% CI: 0.35-1.54)

Conclusion: Our findings support the concept that HIV infection was not a risk factor to increase the severity and risk of death among COVID-19 infected patients.

Keywords: COVID-19, HIV, Severity

Conflicts of Interest: Although Mohammad Mehdi Gouya and Parvin Afsar Kazerooni are CDC managers of the Iranian Ministry of Health and Medical Education, the authors declare that they have no conflict of interests.

Funding: The national database for Medical Care Monitoring Center (MCMC).

\section{*This work has been published under CC BY-NC-SA 1.0 license.}

Copyright $\odot$ Iran University of Medical Sciences

Cite this article as: Eybpoosh S, Afshari M, Haghdoost AA, Afsar Kazerooni P, Gouya MM, Tayeri K. Severity and mortality of COVID-19 infection in HIVinfected individuals: Preliminary findings from Iran. Med J Islam Repub Iran. 2021 (10 Mar);35:33. https://doi.org/10.47176/mjiri.35.33

\section{Introduction}

As of November 23, 2020, coronavirus disease 2019

(COVID-19) has caused 59002152 infections and 1393

Corresponding author: Dr Mahdi Afshari, m_afshari@zbmu.ac.ir

1. Department of Epidemiology and Biostatistics, Research Centre for Emerging and Reemerging Infectious Diseases, Pasteur Institute of Iran, Tehran, Iran

2. Pediatric Gastroenterology and Hepatology Research Center, Zabol University of Medical Sciences, Zabol, Iran

3. HIV/STI Surveillance Research Center, and WHO Collaborating Center for HIV Surveillance, Institute for Futures Studies in Health, Kerman University of Medical Sciences, Kerman, Iran

4. HIV/AIDS Control Office, Center for Communicable Disease, Ministry of Health, Tehran, Iran

5. Center for Disease Control, Ministry of Health and Medical Education, Tehran, Iran

6. Iranian Research Center of HIV/AIDS, Iranian Institute for Reduction of High Risk Behaviors, Tehran University of Medical Sciences, Tehran, Iran $\uparrow$ What is "already known" in this topic:

Higher mortality due to COVID-19 is reported among some immunocompromised patients. The association between immunosuppression due to HIV infection and severity of COVID-19 infection is not widely understood.

\section{$\rightarrow$ What this article adds:}

Analyzing the data from a relatively large sample of COVID19 suspected and confirmed cases showed no significant association between HIV infection and COVID-19 incidence, severity, and fatality rate in Iran. 
879 deaths, resulting in a ca se fatality rate (CFR) of approximately $2.4 \%$, which is higher that the $1 \%$ CFR reported for influenza. The first confirmed COVID-19 cases in Iran were reported in February 19, 2020. By November 23, 2020, Iran's surveillance system has detected 854361 confirmed cases, 13053 of which have died, resulting in a CFR of $1.5 \%$ (1).

The immunodeficiency caused by chronic HIV infection increases the risk of coinfection with other pathogens. In addition, the administration of combination antiretroviral therapy (cART) for HIV coinfected individuals does not always reinstate the pathogen-specific immune response to normal levels. A significant morbidity and mortality in $\mathrm{HIV}$-infected individuals is well known to be caused by serious coinfections with Mycobacterium tuberculosis, hepatitis $\mathrm{B}$ and $\mathrm{C}$ viruses, Cryptococcus spp, parasites, such as Plasmodium spp. The immunosuppressive effect of HIV as well as steroids may also lead to disseminated viral infection caused by coronavirus disease 2019 (COVID-19). However, information on the incidence, severity, and mortality of COVID-19 pneumonia in patients with a low CD4 count due to HIV is lacking.

On the other hand, accumulating evidence suggests that a subgroup of COVID-19 patients may develop a cytokine storm syndrome, characterized by elevated serum levels of interlukines (IL-1 $\beta$, IL-2, IL-7-10, IL-17, G-CSF, GMCSF, interfrons $\alpha$ and $\gamma$, IP10, MCP1, and MIP 1A, and 1B) [1].The syndrom is associated with failure in acute respiratory distress syndrome (ARDS) and failure in lung, heart, liver, and kidney function $(2,3)$. Based on these findings, potentials of immunosuppressant medications have been dicussed for the management of COVID-19 cases with hyperinflamation. Albeit, the area still needs further research. In HIV-infected individuals, disturbances in the regulation of cytokine expression may influence the way these patients respond to the infection with the coronavirus.

Understanding the complex interaction between HIV, coronavirus infection, and the host immune response is central to developing new strategies for optimal management of COVID-19 in HIV-infected patients. The knowledge would be also benefical in better understanding the pathogenesis mechanism of COVID-19 in the human body, and the role of human immune response in disease severity, progression, and outcome. This study aims to investigate the course of COVID-19 infection in HIV-infected individuals by characterizing COVID-19 incidence, clinical presentation, severity, and mortality in HIV-infected patients as compared to HIV-negative COVID cases in Iran. Since the study has been performed in the early stage of the COVID-19 pandemic, few similar evidences have been published in this regard, especially in the study area indicating the novelty of the study.

\section{Methods}

\section{Study Design and Participants}

This was a retrospective cohort study on COVID-19 suspected and confirmed cases hospitlized in Iran between Febuary 19 (epidemic onset date) and April 8, 2020, whose medical information was registered in the national database for Medical Care Monitoring Center (MCMC). We used the patinet information in the MCMC database to retrospectively recruit all COVID-19 suspected and confirmed cases and follow their records from admittion to death/discharge. HIV status was recorded in the database based on patients' self report.

The ethics committee of Zabol University of Medical Sciences approved the study protocol (IR.ZBMU.REC. 1399.059). All data were treated anonymously. Because the study was conducted on the medical records, the requirement for informed consent was waived by the ethics committee.

\section{Definitions}

Based on the national case definition, suspected cases included all SARI cases with: (i) history of dry cough, chills, or sore throat with shortness of breath and with or without fever that could not be justified by other etiological factors; or (ii) fever or respiratory symptoms (with any levels of severity) that had been in close contact with confirmed COVID-19 case(s), 14 days prior to the symptom onset.

COVID-19 confirmed cases included those with a positive polymerase chain reaction (PCR) test result for the SARS-CoV-2 virus based on patients' nasopharyngyal samples. Outpatients were not included in this study due to limited access to the information of this group at the time of the study.

\section{Data Collection}

Primary data regarding demographic and clinical characteristics as well as outcomes of the patients were selected and collected from the MCMC database. The following information was extracted and analysed for each patient: age, gender, medical history, COVID-19-related exposure history, COVID-19 associated sign and symptoms, severity assessment on admission according to the GlascowComa Scale situation, need for intubation and hypoxemia) and laboratory findings, all of which were available in the MCMC database.

\section{Statistical Analysis}

Categorical variables were compared between HIVpositive/negative patients as well as COVID-19 positive/negative cases using chi-square test as well as comparision of the $95 \%$ confidence intrvals. Demographic and clinical variables with significantly different distributions between the 2 groups were entered into the regression models as potential confounders. The association between HIV infection and the onset of COVID-19 infection was assessed in crude and multivariable logistic regression models. The association between coinfection with HIV and COVID-19 clinical manifestations was assessed using univariable and multivariable logistic regression models. To evaluate the effect of HIV infection on the prognosis of the COVID-19 infection, the Cox proportional hazard regression model was used. In this model, death was considered as the main outcome. The "time to death" variable was calculated as the time between the hospital admission and death. Patients were determined as censored if death 
did not occur until April 8 (the perceived time of the discharge of the last record in our dataset). Potential confounders were added to the multivariable models if they had significant or borderline significant associations with the outcome variable in the univariable models. To investigate the interaction between COVID-19 and HIV infection, all the above analyses were performed for confirmed and SARI cases separately and the force of the associations were compared between groups. All data were analysed in Stata software version 14. $\mathrm{P}$ values $<0.05$ were considered statistically significant, while $\mathrm{P}$ values between 0.05 and 0.10 were considered as borderline significant.

\section{Results}

A total of 122206 COVID-19 SARI cases were analysed, of which $65422(53.5 \%)$ were male. Participants' mean (SD) age was 52.1 (19.8) years, with a minimum of less than 1 year and maximum of 91 years.

A total of $90(0.07 \%)$ SARI cases were HIV-positive. The probablity of PCR testing for COVID-19 was not significantly different between HIV-positive and HIVnegative individuals $\left(50.0 \%\right.$ vs $54.3 \%$ respectively, $\mathrm{P}_{\text {Chi- }}$ square $=0.411)$. It means that HIV-positive individuals had been considered and investigated for COVID-19 infection almost the same as the healthy population. COVID-19 test result was positive in $25 \%$ of HIV-negative and $15 \%$ of
HIV-positive SARI cases, with no overlaps in their 95\% CIs, indicating a significant difference between the 2 groups in this regard (Table 1).

COVID-19 suspected patients without comorbidities were more likely to not be tested compared to those with at least 1 comorbidity $(\mathrm{p}<0.001)$. This difference, however, was not statistically significant in HIV-positive patients $(\mathrm{p}=0.511$; Table 2$)$.

There was no statisticaly significant difference between HIV-positive and HIV-negative suspected cases in age $\left(\mathrm{P}_{\mathrm{t}}\right.$ test $=0.750)$, gender $\left(\mathrm{P}_{\text {Chi-square }}=0.887\right)$, and nationality $\left(\mathrm{P}_{\text {Chi-square }}=0.202\right)$. The history of cardiovascular diseases $\left(\mathrm{P}_{\text {Chi-square }}=0.181\right)$, diabetes mellitus $\left(\mathrm{P}_{\text {Chi-square }}=0.245\right)$, and chronic obstructive pulmonary disease $\left(\mathrm{P}_{\text {Chi-square }}=\right.$ 0.632 ) also was not significantly different between the 2 groups. Other comorbidities, however, were significantly more prevalent among HIV-positive suspected cases rather than HIV negative ones.

COVID-19 confirmed cases were significantly older than patients whose PCR test turned negative $(\mathrm{p}<0.001)$. In addition, the frequency of foreigners $(p=0.003)$ and those with at least 1 chronic noncommunicable disease $(p<0.001)$ was significantly lower than that among PCR negative patients. Suspected cases were significantly younger $(\mathrm{p}<0.001)$ than SARI patients and had lower comorbidities $(p<0.001)$ than the others (Table 3$)$.

Table 1. Probability of COVID-19 Testing and Positive Test Result in HIV-Positive and HIV-Negative Patients

\begin{tabular}{|c|c|c|c|c|c|}
\hline \multirow[b]{3}{*}{ COVID-19 Test Results } & \multicolumn{4}{|c|}{ COVID-19 SARI* Cases } & \multirow[b]{3}{*}{$\mathrm{p}^{\S}$} \\
\hline & \multicolumn{2}{|c|}{ HIV-Negatives } & \multicolumn{2}{|c|}{ HIV-Positives } & \\
\hline & $\mathrm{n}(\%)$ & $95 \% \mathrm{CI}$ & $\mathrm{n}(\%)$ & $95 \% \mathrm{CI}$ & \\
\hline Negative & $35,601(29.2)$ & $28.9,29.4$ & $31(34.4)$ & $24.6,44.3$ & 0.104 \\
\hline Positive ${ }^{¥}$ & $30,731(25.2)$ & $24.9,25.4$ & $14(15.6)$ & $8.1,23.1$ & \\
\hline Not tested & $55,784(45.7)$ & $45.4,46.0$ & $45(50.0)$ & $39.7,60.3$ & \\
\hline Total & $122,116(100.0)$ & & $90(100.0)$ & & \\
\hline
\end{tabular}

Table 2. Probability of COVID-19 Testing and Positive Test Result in HIV-Positive and Negative Patients With and Without Comorbidity

\begin{tabular}{lcc}
\hline Groups & No Comorbidty & At Least 1 Comorbidity \\
\hline & $\mathrm{n}(\%)$ & $\mathrm{n}(\%)$ \\
\hline HIV negativepatients & & \\
Negative & $25,897^{\mathrm{a}}(24.1)$ & $9,704^{\mathrm{b}}(31.8)$ \\
Positive & $23,383^{\mathrm{a}}(21.8)$ & $7,348^{\mathrm{b}}(24.1)$ \\
Not tested & $45,432^{\mathrm{a}}(42.3)$ & $10,352^{\mathrm{b}}(34.0)$ \\
HIV positive patients & $17(34.7)$ & $14(30.4)$ \\
Negative & $8(16.3)$ & $6(13.0)$ \\
Positive & $23(46.9)$ & $22(47.8)$ \\
Not Tested & & 0.511 \\
\hline
\end{tabular}

${ }^{\mathrm{a} \& \mathrm{~b}}$ Similar superscript letters indicate non-significant difference between the proportions within the rows.

Table 3. Demographic and Clinical Characteristics of SARI and Suspected Cases

\begin{tabular}{|c|c|c|c|c|c|c|}
\hline \multirow[b]{2}{*}{ Baseline charcteristics } & \multicolumn{2}{|c|}{ COVID-19 Suspected Cases } & \multicolumn{4}{|c|}{ SARI cases } \\
\hline & $\begin{array}{c}\text { PCR Negatives } \\
\mathrm{n}(\%)\end{array}$ & $\begin{array}{c}\text { confirmed Cases } \\
\mathrm{n}(\%)\end{array}$ & $\mathrm{p}$ & $\begin{array}{c}\text { Suspected Cases } \\
\mathrm{n}(\%)\end{array}$ & $\begin{array}{c}\text { Other SARI Cases cases } \\
\mathrm{n}(\%)\end{array}$ & $\mathrm{p}$ \\
\hline $\mathrm{Age}_{\text {Mean (SD) }}$ & $52.94(21.43)$ & $56.18(17.56)$ & 0.0001 & $49.33(19.54)$ & $54.70(19.34)$ & $<0.001$ \\
\hline \multicolumn{7}{|l|}{ Gender } \\
\hline Female & $16,706(46.88)$ & $13,570(44.14)$ & $<0.001$ & $26,508(47.48)$ & $37,443(45.56)$ & $<0.001$ \\
\hline Male & $18,926(53.12)$ & $17,175(55.86)$ & & $29,321(52.52)$ & $44,736(54.44)$ & \\
\hline \multicolumn{7}{|l|}{ Nationality } \\
\hline Iranian & $34,396(96.53)$ & 29,805 (96.94) & 0.003 & $53,134(95.17)$ & $78,923(96.04)$ & $<0.001$ \\
\hline Foreigner & $1,236(3.47)$ & $940(3.06)$ & & $2,695(4.83)$ & $3,256(3.96)$ & \\
\hline Comorbidity* & & & $<0.001$ & & & \\
\hline No chronic disease & $25,914(72.73)$ & $23,391(76.08)$ & & $45,455(81.42)$ & $62,021(75.47)$ & $<0.001$ \\
\hline At least one chronic disease & $9,718(27.27)$ & $7,354(23.92)$ & & $10,374(18.58)$ & $20,158(24.53)$ & \\
\hline
\end{tabular}

Cardiovascular; diabetes; cancer; COPD; renal; hamatologic and immunodeficiency disorders. 


\begin{tabular}{|c|c|c|c|c|c|c|c|}
\hline Odds of HIV Infection & & $\begin{array}{c}\text { HIV Negative } \\
\mathrm{n}(\%)\end{array}$ & $\begin{array}{c}\text { HIV Positive } \\
\text { N }(\%)\end{array}$ & $\mathrm{p}$ & $\begin{array}{c}\text { Adjusted* } \\
\text { OR }\end{array}$ & $\bar{p}$ & $95 \% \mathrm{CI}$ \\
\hline Odds of having HIV infection & Negative PCR $(n=35,632)$ & $35,601(99.91 \%)$ & $31(0.09 \%)$ & 0.041 & 1 & & 1 \\
\hline in confirmed cases vs others & Confirmed $(n=30,754)$ & $30,731(99.95 \%)$ & $14(0.05 \%)$ & & 0.54 & 0.060 & $0.29,1.02$ \\
\hline Odds of having HIV infection & Others $(\mathrm{n}=55,829)$ & $55,784(99.92 \%)$ & $45(0.08 \%)$ & 0.170 & 1 & & 1 \\
\hline in suspected cases vs others & Suspected $(n=82,179)$ & $82,129(99.94 \%)$ & $50(0.06 \%)$ & & 0.68 & 0.061 & $0.45,1.02$ \\
\hline
\end{tabular}

Controlled for the effect of comorbidities, gender, age, nationality.

Table 4 shows that HIV infection decreased the odds of confirmed COVID-19 infection as of $46 \%$ (adjusted OR= $0.54 ; p=0.060)$, while the odds of being a suspected case was reduced as of $32 \%$ (adusted $\mathrm{OR}=0.68 ; \mathrm{p}=0.061$ ).

The odds ratios were also estimated in 2 periods: the beginning of the epidemic in Iran (winter 2019) and the next months (Spring 2020). These ratios were estimated as 0.5 (95\% CI: $0.3-1.1)$ and 0.6 (95\% CI: 0.2- 1.8), respectively (Table 5).

Crude and adjusted odds ratios for developing critical signs and symptoms of COVID-19 in HIV-positives rather than HIV-negatives were estimated among SARI, suspected and confirmed cases. The odds of low values of Glasgow coma scale (GCS) was higher in cases with HIVCOVID-19 coinfection rather than COVID-19 single infections. However, in suspected and SARI cases, HIV infection caused respectively $33 \%$ and $1 \%$ decreased odds of having low GCS. HIV-positives also had a higher chance of experiencing intubation in all 3 groups. However, non of these associations were statistically significant. On the other hand, HIV-positive cases showed a $40 \%$ and $19 \%$ lower chance of having low $\mathrm{O}_{2}$ saturation, respectively, among confirmed cases and suspected/SARI patients. These associations were not statistcally significant either. The hazard ratios for death in HIV-positive COVID-19 confirmed cases was 0.33 (95\% CI: 0.05 , 2.32). This value was 0.81 (95\% CI: $0.33,1.94)$ and 0.73 (95\% CI: $0.35,1.54)$ among COVID-19 suspected and SARI cases who were HIV-positive, respectively. Although HIV infection reduced the risk of death among con- firmed cases more than suspected and SARI patients, the associations was not statistically significant (Table 6).

\section{Discussion}

Our study showed that the probability of a positive COVID-19 PCR test result was lower among HIVpositives than negatives. This might be due to the surveillance bias, which here means testing HIV-positive cases more frequently, regardless of their eligibility for COVID19 testing. Because of their special clinical conditions, we expected that HIV-positive cases were more tested. However, frequency of HIV testing among HIV-positives and the rest of the samples were almost the same, with HIVpositives being less probable to have positive results.

We also needed to investigate and rull out selection bias, which here means refering and admitting HIV-positive patients to the hospitals more frequently than the rest of the population. To rule out this bias, we compared the proportion of HIV-positive cases in our study sample with that in the total population. By April 5, 2020, about 0.07 of our study sample were HIV-positve, while the corresponding figure for the general population was also 0.07 (personal communication with the Ministry of Health). In addition, investigating the results of the online active screening of the poulation with the current systems of the Ministry of Health can show us an estimate of this proportion.

The high self protection of HIV-positive patients during the COVID-19 pandemic might be considered as a reason for lower risk of infection in this group. If so, the effect of

Table 5. The Association Between HIV Infection and Developing COVID-19 Infection in Different Periods

\begin{tabular}{|c|c|c|c|c|}
\hline Odds Ratio (P Value) & \multicolumn{4}{|c|}{ 95\% Confidence Intervals } \\
\hline Winter 2020 & \multicolumn{4}{|c|}{$0.26,1.15$} \\
\hline Spring 2020 & \multicolumn{4}{|c|}{$0.17,1.82$} \\
\hline \multicolumn{5}{|c|}{$\begin{array}{l}\text { Table 6. Crude and Adjusted Odds Ratios(OR) for HIV Infection and Clinical Presentation and Prognosis of Suspected/Confirmed COVID-1 } \\
\text { Infection }\end{array}$} \\
\hline & & Adjusted* OR (HR) & P Value & $95 \% \mathrm{CI}$ \\
\hline \multirow{3}{*}{ Odds of having low GCS in HIV-POSITIVE vs HIV- Negative } & Confirmed cases & 1.70 & .616 & $0.21,13.73$ \\
\hline & Suspected cases & 0.68 & .594 & $0.16,2.82$ \\
\hline & All SARI cases & 0.99 & .984 & $0.40,2.47$ \\
\hline \multirow{3}{*}{ Odds of need for intubation in HIV-POSITIVE vs HIV- Negative } & Confirmed cases & 1.70 & .494 & $0.40,7.86$ \\
\hline & Suspected cases & 1.39 & .453 & $0.59,3.29$ \\
\hline & All SARI cases & 1.02 & .950 & $0.47,2.23$ \\
\hline \multirow[t]{3}{*}{ Odds of having low PO2 in HIV-POSITIVE vs HIV- Negative } & Confirmed cases & 0.60 & .370 & $0.20,1.82$ \\
\hline & Suspected cases & 0.81 & .460 & $0.45,1.43$ \\
\hline & All SARI cases & 0.81 & .330 & $0.53,1.23$ \\
\hline \multirow[t]{3}{*}{ Hazard of developing death in HIV-POSITIVE vs HIV- Negative } & Confirmed cases & 0.33 & .264 & $0.05,2.32$ \\
\hline & Suspected cases & 0.81 & .632 & $0.33,1.94$ \\
\hline & All SARI cases & 0.73 & .412 & $0.35,1.54$ \\
\hline
\end{tabular}

* Controlled for the effect of comorbidities, age and pregnancy. 
HIV-COVID-19 comorbidity is expected to vary over time. To assess the effect of the time in testing the patients, the association between HIV infection and the incidence of COVID-19 infection was estimated for patients referrals in the 2 periods: at the beginning and the ongoing phase of the COVID-19 epidemic in Iran. In both periods, HIV infection status (positive/negative) was not associated with COVID-19 infection, suggesting no reverse associations between HIV and COVID-19 infections.

We also comapred COVID-19 testing rate between HIV-positive and the rest of the sample while taking other comorbidities into account. The results showed that COVID-19 testing among HIV-positives was performed regardless of their underline medical conditions, which can be in favor of bias toward more testing of HIV cases. In HIV-negatives, however, having comorbidities other than HIV did not predict COVID-19 testing. This suggests that HIV-positive patients were tested for COVID-19 just because of having HIV infection.

To investigate the effect of HIV infection on the risk of COVID-19, regression models controlling for the effect of potential confounders, such as age, and chronic comorbidities, were applied. Although being HIV-positive reduced the odds of COVID-19 infection, the observed association was statistically borderline significant, indicating that HIV-positive patients had no higher risk of developing COVID-19 infection compared to the rest of our sample. In addition, to evaluate any probable interaction between HIV and COVID-19 infection, the mentioned statistical models were applied for all SARI cases and the effects of HIV infection on developing confirmed and suspected cases were compared. Results showed that although the force of association was stronger for confirmed cases, both effect measures were nonsignificant, indicating no evidence of interaction. This was in contrast with the findings reported by Del Amo et al (4). They reported a lower risk of COVID-19 incidence among HIV positive patients than in the general population. Although the number of HIV-positive cases in the Del Amo et al's study was higher than ours, they did not include comparison group as we did (they only studied HIV positive patients). For comparison, they compared their results with external estimates in the general population.

One of the main goals of the present study was to investigate the association between HIV infection and COVID19 severity. Results of multivariable models showed that having HIV infection may not affect the severity of signs, symptoms, and paraclinical findings of the COVID-19 positive patients.

We also investigated the association between HIV infection and prognosis of COVID-19 infection. Of different outcomes eligible for this evaluation, information on patients' death could only be investigated because of the availablity of complete data in this regard. The results of suvival analyses showed that patients with HIV infection had the same survival experience as the rest of our sample. Even after controlling for the effect of the potential confounders, having HIV infection did not change the hazard of death in patients with COVID-19 infection. Although stratum specific associations (for confirmed, suspected and SARI cases) showed a stronger effect of HIV infection on developing severe manifestations and death among COVID-19 confirmed cases than suspect or SARI patients, observed associations were not statistically significant. These comparative results are in favor of no interaction between HIV and COVID-19 infections on developing severe manifestations and poor prognosis. Similarly, Sigel et al found no differences between HIV positive COVID-19 cases and HIV negative ones (5). However, Del Amo et al found higher risk of death among HIVpositive patients than the general population (4). The risk of day 28 mortality was also increased among COVID-19 patients with HIV infection in the United Kingdom (6). The similarity of the latter study with our study is the relatively low number of HIV positive cases included in the studies. Moreover, that study was performed among COVID-19 patients admitted to the hospitals. In addition, HIV infection caused more than 2 folds increase in the risk of mortality among COVID-19 patients in SubSaharan Africa (7).

These associations might be prone to deletion bias. For example, during the first months of the epidemic in Iran, just patients with severe signs and symptoms were being tested for COVID-19. Therefore, the equal clinical manifestations in HIV-positive and negative cases can be due to such bias. In addition, due to surveillance bias, HIVpositive patients might be referred for testing more frequently than the other patients, leading to lower signs and symptoms as well as better prognosis. Therefore, the 2 variables that were needed to be considered in our analyses were the onset date of the symptomes as well as the diagnostic delay. Unfortunately, we did not have access to this information, which calls the need for further research in this area.

The lack of information regarding the biomarlers of HIV-infection clinical progress status, such as CD4 counts and adherence to antirettroviral therapy, limited our ability to investigate the role of these variables in the observed associations. In addition, we did not access other outcome variables, such as duration of the disese, time of intubation/extubation, time of fever supression, etc., which warant further research in this area.

\section{Conclusion}

Our study did not reveal any significant association between HIV infection and COVID-19 onset, severity and prognosis/mortality, with HIV-positive cases having slightly lower probability of death due to COVID-19 than HIV-negatives. Further longitudinal cohorts are recommended to determine the exact interactions between these 2 viral infections.

\section{Conflict of Interests}

The authors declare that they have no competing interests.

\section{References}

1. WorldOmeters. COVID-19 Coronavirus Pandemic/Iran. WorldOmeters; [updated January 21, 2021; cited January 21, 2021]. Available from: https://www.worldometers.info/ 
coronavirus/country/iran

2.Huang C, Wang Y, Li X, Ren L, Zhao J, Hu Y, et al. Clinical features of patients infected with 2019 novel coronavirus in Wuhan, China. Lancet. 2020;395(10223):497-506.

3.Xu Z, Shi L, Wang Y, Zhang J, Huang L, Zhang C, et al. Pathological findings of COVID-19 associated with acute respiratory distress syndrome. Lancet Resp Med. 2020;8(4):420-2.

4. Del Amo J, Polo R, Moreno S, Diaz A, Martinez E, Arribas J, et al. Incidence and Severity of COVID-19 in HIV-Positive Persons Receiving Antiretroviral Therapy. Ann Intern Med. 2020.

5. Sigel K, Swartz T, Golden E, Paranjpe I, Somani S, Richter F, et al. Coronavirus 2019 and people Living With Human Immunodeficiency Virus: Outcomes for Hospitalized Patients in New York City. Clin Inf Dis. 2020:1-6.

6. Geretti AM, Stockdale AJ, Kelly S, Cevik M, Collins S, Waters L, et al. Outcomes of COVID-19 related hospitalization among people with HIV in the ISARIC WHO Clinical Characterization Protocol (UK): a prospective observational study. Clin infect Dis. In press 2020.

7. Boulle A, Davies MA, Hussey H, Ismail M, Morden E, Vundle Z, et al. Risk factors for COVID-19 death in a population cohort study from the Western Cape Province, South Africa. Clin infect Dis. In press 2020. 